\title{
Grundrechte als Schranken der Vertragsfreiheit in europäischen Privatrechtssystemen
}

\begin{abstract}
Summary
This contribution explores the application of basic rights to contractual freedom in European legal systems given the background of a general tendency to constitutionalization and materialization of private law. Examples of methodological instruments and forms of justification are analyzed which play a role in the application of basic rights in relation to contraction freedom. This is done using the examples of Italy's and France's judicatures, where basic rights can lead to a limitation of contractual law with differing consequences. A methodological reflection is added to these results: If private law continues to be increasingly put in the sphere of influence of basic rights and higherranking principles, this will result in an increase in the importance of weighing these principles against one another. In order to look into the consequences of such changes, which could even be of importance in view of a future unifying effect of European basic rights, the specific role of basic rights in the methodological rules of European private law systems must be examined. A historical-comparative reflection of principle-oriented private law is needed.
\end{abstract}

\section{Résumé}

Cette contribution examine l'application des droits fondamentaux à la liberté contractuelle dans les systèmes juridiques européens, compte tenu de la tendance généralisée à la constitutionnalisation et à la matérialisation du droit privé. Elle analyse divers exemples d'instruments méthodologiques et de formes de justification jouant un rôle dans l'application des droits fondamentaux vis-à-vis de la liberté contractuelle. Ce faisant, elle s'appuie sur les exemples des juridictions italienne et française, au sein desquelles les droits fondamentaux peuvent engendrer une restriction du droit contractuel, avec diverses conséquences. Une réflexion méthodologique vient compléter ces résultats : si le droit privé continue à être de plus en plus fréquemment intégré à la sphère d'influence des droits fondamentaux et des principes de rang supérieur, il deviendra d'autant plus important d'évaluer ces principes les uns par rapport aux autres. Afin d'examiner les conséquences de ces changements, qui pourraient même s'avérer essentiels dans la perspective d'un futur effet fédérateur des droits fondamentaux européens, il convient d'étudier le rôle spécifique que jouent les droits fondamentaux dans les règles méthodologiques des systèmes de droit privé européens. Il est également essentiel de mener une réflexion comparative historique du droit privé reposant sur des principes. 


\section{Einleitung: Konstitutionalisierung und Materialisierung des Vertrags- rechts}

Das Verhältnis von Grundrechten und Privatrecht war stets ein wichtiges Thema. ${ }^{1}$ In Deutschland denkt man dabei unweigerlich an die Geschichte des Grundgesetzes und der Orientierung des Privatrechts an dessen übergeordneten Prinzipien. Bislang spielte für das deutsche Privatrecht die nationale Verfassung eine wichtige Rolle. Besonders aktuell ist zur Zeit die Reflexion über Grundrechte und Privatrecht vor dem Hintergrund supranationaler, vor allem europäischer Zusammenhänge. Impulse gehen von mehreren Ebenen aus. $\mathrm{Zu}$ konstatieren ist zunächst ein allgemeiner Bedeutungszuwachs von grundlegenden Regeln und Prinzipien in einem immer sichtbarer werdenden Bereich „europäischen Rechts“ im weiteren Sinn. Am 1. Dezember 2009 hat die europäische Grundrechtecharta Rechtsverbindlichkeit erlangt. Ihre Konsequenzen für die Privatrechtsordnungen europäischer Staaten hat der Gesetzgeber (wohl bewusst) nicht eindeutig thematisiert. ${ }^{2}$ Sie kann daher vorerst für die Praxis weder als neue Wirbelsäule eines grundsatzorientierten europäischen Privatrechts, noch als vorläufiger Abschluss einer Entwicklung zur Manifestierung höherrangigen Rechts gelten. Ungeachtet dessen wird die Reflexion über ,,allgemeine Rechtsgrundsätze“3 immer wichtiger, auch im Sinne angelsächsischer Vorstellungen von ,general principles of law“. ${ }^{4}$ Unter dem Eindruck der Rechtsprechung des Europäischen Gerichtshofs in Bezug auf Altersdiskriminierung (Mangold-Rechtsprechung) ${ }^{5}$ wurde eine teilweise Verdrängung deutschen Dienstvertragsrechts durch europäische Grundrechte offensichtlich, ${ }^{6}$ die in der deut-

1 Probevortrag zur Habilitation am Fachbereich Rechtswissenschaft der Goethe-Universität Frankfurt am Main vom 1. Februar 2012. Die Vortragsform wurde im wesentlichen beibehalten und mit Nachweisen versehen, die jedoch keinen Anspruch auf Vollständigkeit erheben.

2 Jürgen Meyer, Charta der Grundrechte der Europäischen Union, 3. Aufl. Baden-Baden 2011, Art. 51, Rn. 31. Art. 51 GRCh bezieht den Anwendungsbereich auf Organe der Union und der Mitgliedstaaten, sofern sie das Recht der Union „durchführen“. Eine Bindung von Privatpersonen scheidet damit aus, nicht aber eine Ausstrahlung auf Entscheidungen nationaler Gerichte, sofern diese Unionsrecht anwenden, etwa bei richtlinenkonformer Auslegung. Hans D. Jarass, Charta der Grundrechte der Europäischen Union. Kommentar. München 2010, Art. 51, Rn. 24-27. Zu aktuellen Beispielen aus dem Bereich des gewerblichen Rechtsschutzes: Benjamin Raue, Die Verdrängung deutscher durch europäische Grundrechte im gewerblichen Rechtsschutz und Urheberrecht, GRUR Int 2012, S. 402-410. Zu privatrechtlichen Regulierungsfunktionen der Charta: Christoph Busch, Fundamental Rights and Private Law in EU Member States, in: ders./Hans Schulte Nölke (Hrsg.), EU Compendium Fundamental Rights and Private Law. A practical tool for judges, München 2011, S. 1-25 (3-7). Siehe auch Christoph Busch, Europäischer Grundrechtsschutz im Privatrecht nach Lissabon: Die EU-Grundrechtecharta als neuer Prüfungsmaßstab für Umsetzungsgesetze, DRiZ 2010, S. 63-66.

3 Ausführlich Axel Metzger, Extra legem, intra ius: Allgemeine Rechtsgrundsätze im Europäischen Privatrecht, Tübingen 2009, v.a. S. $221 \mathrm{ff}$.

4 Siehe etwa Anthony Arnull, What is a general principle of EU-Law?, in: Rita de la Feria/Stefan Vogenauer (Hrsg.), Prohibition of Abuse of Law, Oxford 2011, S. 7-23 (16).

5 EuGH (Große Kammer), Urteil vom 22. 11. 2005 - C 144/04 Werner Mangold/Rüdiger Helm = NZA 2005, S. 1354-1348; EuGH, Urteil vom 19. 1. 2010 - C-555/07 Seda Kücükdeveci/ Swedex GmbH \& Co. KG = NZA, 2010, S. 85-89 (als Fortsetzung des Mangold-Urteils).

6 Siehe z.B. Jobst-Hubertus Bauer/Andreas von Medem, Kücükdeveci = Mangold hoch zwei? Europäische Grundrechte verdrängen deutsches Arbeitsrecht, ZIP 2010, S. 449-457. 
schen Literatur überwiegend skeptische Reaktionen auslöste. ${ }^{7}$ Nicht von der Hand zu weisen war eine vom Europäischen Gerichtshof $(\mathrm{EuGH})$ vorgenommene Bekräftigung primär- und sekundärrechtlicher Diskriminierungsverbote. Das ließ die Frage nach einem allgemeinen europäischen Grundsatz der Gleichbehandlung als Rechtsprinzip entstehen, auch jenseits des Arbeitssektors. ${ }^{8}$ Die Schrankenfunktion des Gleichbehandlungsprinzips für die Vertragsfreiheit in den Mitgliedstaaten war offensichtlich. ${ }^{9}$ Neben der richterrechtlichen Fortbildung europäischer Grundrechte aus Quellen des Europarechts, haben auch Grundrechtskataloge wie die Europäische Menschenrechtskonvention Auswirkungen auf nationale Privatrechtssysteme. ${ }^{10}$ Aus deutscher Sicht denkt man hier vor allem an die Entwicklung des Persönlichkeitsrechts im Zuge der sog. CarolineEntscheidungen, bei der deutsches Richterrecht zu Presseveröffentlichungen von Bildmaterial über Prominente an Maßstäben der Art. 8 und 10 EMRK gemessen wurde. ${ }^{11}$ Deren Spuren im deutschen Deliktsrecht sind nun nicht mehr zu übersehen. ${ }^{12}$

Auf europarechtlicher Ebene ist die Frage nach einem „sozialen“, vielleicht in Grundrechten wurzelnden Korrektiv für die Grundfreiheiten ${ }^{13}$ seit längerer Zeit aktuell. Solche Überlegungen berühren auch das Privatrecht. Im Jahr 2004 wurde in einem Manifest europäischer Rechtswissenschaftler zu „Social Justice in European Contract Law“ die europäische Grundrechtecharta bei entsprechender Konkretisierung als Leitbild sozialer Gerechtigkeit im Vertragsrecht hervorgehoben. ${ }^{14}$ Sollten im Zuge der aktuellen Krise wirtschaftspolitische Kompetenzen der Union erweitert werden, wird die Frage nach einer sozialen Dimension der politischen Gestaltung von Märkten auf europäischer Ebene noch nachdrücklicher gestellt werden. Dieser Problemkomplex, dessen Ausmaße hier nur annähernd skizziert worden sind, lässt sich mit zwei allgemeinen Schlagworten kennzeichnen: Es geht um Materialisierung und Konstitutionalisierung des Privatrechts.

Beide Begriffe haben, auch wegen ihrer Wertungs- und Assoziationsoffenheit, Karriere gemacht: „Konstitutionalisierung“ vor allem im Bereich des öffentlichen Rechts

7 Nachweise bei Norbert Reich, „Mangold“ und kein Ende - oder doch?, EuZW 2007, S. 198 f. Vgl. aktuell Jan Lüttringhaus, Die Vertragsfreiheit kommt in Brüssel unter die Räder, Frankfurter Allgemeine Zeitung 05/04/2011, S. 19.

8 Vgl. auch Mathias Habersack/Tobias Tröger, „Ihr naht euch wieder, schwankende Gestalten...", Zur Frage eines europarechtlichen Gleichbehandlungsgrundsatzes beim Anteilshandel, NZG 2010, S. 1-7.

9 Axel Metzger, Allgemeine Rechtsgrundsätze in Europa - dargestellt am Beispiel des Gleichbehandlungsgrundsatzes, RabelsZ 78 (2011), S. 845-881, dort auch zur Beschränkung der Vertragsfreiheit, S. $877 \mathrm{ff}$.

10 Vergleichend bzgl. mehrerer Länder Günter Hager, Rechtsmethoden in Europa, Tübingen 2009, S. 222-249, vgl. auch Helen Keller/Alec Stone-Sweet (Hrsg.), A Europe of Rights. The Impact of the ECHR on National Legal Systems, Oxford 2008.

11 Überblick bei Wolfgang Hoffmann-Riem, Die Caroline II-Entscheidung des BVerfG - Ein Zwischenschritt bei der Konkretisierung des Kooperationsverhältnisses zwischen den verschiedenen betroffenen Gerichten, NJW 2009, S. 20-26.

12 Vgl. BGH Urteil vom 14.10.2008 - VI ZR 271/06 = NJW 2009, S. 754-756.

13 Zu letzterem Stefan Kadelbach/Niels Petersen, Europäische Grundrechte als Schranken der Grundfreiheiten, EuGRZ 2003, 693-698.

14 Study Group on European Private Law, Social Justice in European Contract Law: a Manifesto, European Law Journal 10 (2004), S. 653-674 (673). 
im weitesten Sinne, ${ }^{15}$ „Materialisierung“ vor allem im Bereich des Privatrechts - sofern man die Differenzierung beider Bereiche noch für sinnvoll hält. ${ }^{16}$ Konstitutionalisierung bedeutet dann, mit Blick auf den hier gekennzeichneten Themenbereich, Ausrichtung und Bindung einer Materie an Verfassungsrecht, oder gar Überlagerung oder Verwachsen mit verfassungsrechtlichen Strukturen. ${ }^{17}$ Zumindest bringt Konstitutionalisierung neues Recht hervor, vielleicht auch neue Prinzipien. Erforderlich werden vielleicht sogar neue Methoden.

Materialisierung steht bei vielen Autoren für die Überwindung ,des Formalen' durch ein Zur Geltung Bringen von Inhalten und Gerechtigkeitsanforderungen jenseits einer ,bloß rechtlichen' Definition menschlicher Handlungsspielräume. ${ }^{18}$ Assoziiert wird hiermit oft die Verwirklichung tatsächlicher Ziele und ein an konkreten Bedürfnissen ausgerichtetes Normanwendungsprogramm, das auch eine politische Natur nicht zu verleugnen braucht. Nicht selten besteht ein Wechselwirkungszusammenhang zwischen Konstitutionalisierung - also prinzipieller Orientierung an höherrangigem Recht - und Materialisierung des Privatrechts. Andererseits kann „Materialisierung“, durch Beschränkung von Vertragsfreiheit oder Herstellung von Vertragsgerechtigkeit, ${ }^{19}$ auch privatrechtsimmanent erfolgen, ohne Rückbindung an höherrangiges Recht. Instrumente dazu sind etwa Verbraucherschutznormen ${ }^{20}$ oder das Institut des Rechtsmissbrauchs. ${ }^{21}$ Schwieriger wird die Unterscheidung bei den Generalklauseln, wegen ihrer Scharnierfunktion zwischen Privat- und Verfassungsrecht. Zumindest wenn sie als bloße Einfallstore für Verfassungsrecht fungieren, sind sie nach kontinentalem Verständnis Elemente einer Konstitutionalisierung des Privatrechts. Insgesamt ist die Unterscheidung zwischen Materialisierung mit oder ohne Verfassung ein wichtiges Kriterium, zeigt sie doch stets den Weg zu Alternativen. ${ }^{22}$ Die Frage nach Vertragsgerechtigkeit und der Notwendigkeit von Freiheitsbeschränkungen stellte sich schon lange bevor es Verfassungen und Grundrechtskataloge gab, die bekanntlich eine relativ neue Option sind. Im Folgenden sollen Ansätze zur Analyse der Modalitäten und Möglichkeiten einer Beschränkung privatrechtlicher Freiheit auf europäischer Ebene entwickelt werden.

15 Matthias Knauff, Konstitutionalisierung im inner- und überstaatlichen Recht - Konvergenz oder Divergenz?, ZaöRV 68 (2008), 453-490 (455 f. m.w.N.).

16 Claus-Wilhelm Canaris, Wandlungen des Schuldvertragsrechts - Tendenzen zu seiner „Materialisierung“, AcP Bd. 200 (2000), S. 273-364 (276 ff.).

17 Dem liegt freilich ein kontinentales Rechts- und Verfassungsverständnis zugrunde, das etwa einer vom Common Law geprägten Rechtskultur nicht ohne weiteres entspricht. Siehe Christoph Möllers, Verfassungsgebende Gewalt - Verfassung - Konstitutionalisierung, in: Armin von Bogdandy/Jürgen Bast (Hrsg.), Europäisches Verfassungsrecht, Dordrecht 2009, S. 227-278 (265 ff.).

18 M.w.N. Marietta Auer, Materialisierung, Flexibilisierung und Richterfreiheit, Tübingen 2005, S. 23-45.

19 Zu dieser Differenzierung Canaris, AcP 200 (2000), S. 282, näher dazu auch Auer, Materialisierung, S. 23.

20 Canaris, AcP 200 (2000), S. 343 ff.

21 Dazu europäisch vergleichend Filippo Ranieri, Verbot des Rechtsmissbrauchs und Europäisches Gemeinschaftsprivatrecht, ZEuP 2001, S. 165-177.

22 Vgl. auch Auer, Materialisierung, S. $145 \mathrm{ff}$. 


\section{Beschränkung der Vertragsfreiheit durch Grundrechte als „Materiali- sierung “}

Die soeben umrissene Fragestellung bedarf der Konkretisierung. Materialisierung und Konstitutionalisierung von Privatrecht kann man aus sehr verschiedenen Perspektiven mit unterschiedlichen Schwerpunkten betrachten. Eine europarechtliche Sicht auf das Thema könnte das Unionsprivatrecht in den Blick nehmen und etwa Grundrechtswirkungen bei der Anwendung von Richtlinien erforschen. Hier soll es jedoch um eine rechtsvergleichende Analyse europäischer Privatrechtssysteme gehen, wobei nur einige Schlaglichter auf ausgewählte Länder und Beispiele geworfen werden können. Die Rechtsvergleichung hat es nicht zuletzt mit der Analyse von Grundrechtskulturen- und semantiken verschiedener Privatrechtssysteme zu tun. Für die Konstitutionalisierung des Privatrechts ist Richterrecht ein entscheidender Faktor, nicht nur in Deutschland, mit der prägenden Erfahrung des Lüth-Urteils. ${ }^{23}$ Eine mit „Materialisierung“, also Gerechtigkeitsansprüchen, verbundene Mobilisierung von Grundsätzen höherrangiger Art kann man auch in Frankreich, vor allem aber in Italien beobachten. ${ }^{24}$ Eine wichtige Funktion hat dabei der nationale Grundrechtskatalog. Würde man den Fokus nur auf eine Anwendung europäischer, supranationaler Grundrechte lenken, wäre die Perspektive zu eng. Gerade die Reflexion über eine künftige Tragweise europäischer Grundrechte, oder überhaupt, europäischer Rechtsvereinheitlichung durch Prinzipien, erfordert vergleichende Kenntnisse der bereits bestehenden Rezeptionsgewohnheiten und Funktionen solcher Prinzipien in den nationalen Privatrechtsordnungen. Träger der Grundrechtsrezeption und damit Schrittmacher der Europäisierung sind die Gerichte. ${ }^{25}$ Notwendig ist daher besondere Aufmerksamkeit für Urteile aus verschiedenen Ländern und deren Untersuchung auf grundrechtssensible Bereiche. Letztere soll hier im Vordergrund stehen. Einige aktuelle Studien auf diesem Gebiet sind in ähnlicher Weise vorgegangen. Ein umfangreiches europäisches Netzwerkprojekt befasste sich zwischen 2002 und 2006 mit „Fundamental Rights and Private Law in the European Union“. Die Ergebnisse der empirisch von Grundrechtswirkungen auf das Privatrecht verschiedener Länder ausgehenden Studie liegen seit 2010 vor. ${ }^{26}$ Sie enthält detaillierte Länderberichte über - nicht zuletzt justiziell geprägte - Selbstverständnisse verschiedener Rechtsordnungen in Bezug auf Grundrechte und Privatrecht. ${ }^{27}$ Aktuelle Forschungsimpulse in dieser Richtung gehen von der Europäischen Union aus. ${ }^{28}$ Rechts-

23 BVerfGE 7, 198.

24 Näheres dazu im folgenden Abschnitt.

25 Ausführlich Jürgen Basedow, Nationale Justiz und europäisches Privatrecht - Eine Vernetzungsaufgabe, Heidelberg 2003.

26 Gert Brüggemeier/Aurelia Colombi Ciacchi/Giovanni Comandé (Hrsg.), Fundamental rights and Private Law in the European Union, Bd. 1 und 2, Cambridge 2010.

$27 \mathrm{Zu}$ Leitfragen und Vorgehensweise Brüggemeier/Colombi Ciacchi/ Comandé (Hrsg.), Fundamental rights, Bd. 1, S. 2-7.

28 Christoph Busch/Hans Schulte-Nölke (Hrsg.), EU Compendium Fundamental Rights and Private Law, München 2011. 
vergleichende Monographien sind ebenfalls vorhanden. ${ }^{29}$ Verschiedene Vorgehensweisen sind dabei möglich. Man kann Rechtsgebiete eingrenzen, um dort Konstitutionalisierungen $\mathrm{zu}$ beobachten, etwa nach Auswirkungen der Informationsfreiheit im Mietrecht fragen, oder dem Grundrechtschutz der Familie, beim Nachzug von Ehegatten u.s.w. Im Arbeitsrecht sind Diskriminierungsverbote und Begrenzungen von Direktionsrechten ein Thema. So könnte man eine kontinuierliche Modifikation von Prinzipien, vielleicht sogar Systemrationalitäten, unter dem Einfluss von Grundrechten beobachten. ${ }^{30}$ Man kann auch von einem bestimmten Normkomplex, etwa der EMRK, oder einem dort verankerten Rechtsinstitut, als Exportartikel für verschiedene Rechtssysteme ausgehen. ${ }^{31}$ Hier soll hingegen ein zentrales Strukturprinzip europäischer Privatrechtssysteme in den Vordergrund gestellt werden. Ich möchte mich auf die Vertragsfreiheit konzentrieren und die Frage stellen, wie sie durch Grundrechte begrenzt wird und welche Ergebnisse dabei zutage treten. Dazu habe ich exemplarisch Rechtsprechung aus Italien und Frankreich ausgewählt. Drei leitende Fragestellungen sollen auf die jeweiligen Fallbeispiele bezogen werden, nämlich (1) ob sich Langzeittendenzen einer „Materialisierung" des Vertragsrechts in dem jeweiligen Land abzeichnen und (2) welche methodischen Konsequenzen das hätte. Schließlich soll (3) in einem Ausblick auch auf die rechtskulturelle Bedeutung dieser Konstitutionalisierungen eingegangen werden.

\section{Ausgewählte Konstitutionalisierungsprozesse von Vertragsrecht}

\section{Grundrechtsevolution: Vertragsfreiheit versus Solidarität in Italien}

Für Vergleiche mit Deutschland besonders attraktiv erscheint Italien, denn auch hier ist nach dem 2. Weltkrieg ein Grundrechtskatalog gleich zu Beginn in der Verfassung normiert worden. Art. 2 der Verfassung der italienischen Republik von 1947 lautet:

„,Die Republik anerkennt und gewährleistet die unverletzlichen Rechte des Menschen sowohl als Einzelperson, als auch innerhalb der gesellschaftlichen Gruppen, in denen sich seine Persönlichkeit entfaltet. Sie fordert die Erfüllung der unabdingbaren Pflichten politischer, wirtschaftlicher und sozialer Solidarität ". 32

Besonders bemerkenswert ist der letzte Teil: Die Republik garantiert nicht nur Individualrechte gegenüber dem Staat, sie fordert auch die Einhaltung eines Prinzips, nämlich „solidarietà“. Mit einer Erwähnung von Solidarität an so prominenter Stelle, wird die

29 V.a. Chantal Mak, Fundamental rights in European Contract Law, Aalphen 2008; Olga Cherednychenko, Fundamental rights, Contract Law and the Protection of the Weaker Party, München 2007.

30 Vgl. insgesamt dazu die Ergebnisse der Studie Brüggemeier/Colombi Ciacchi/Comandé (Hrsg.), Fundamental rights, Bd. 2, S. 33 ff, $111 \mathrm{ff}$.

31 So die Vorgehensweise von Hager, Rechtsmethoden, S. $222 \mathrm{ff}$.

32 Übersetzung hier nach Peter Kindler, Einführung in das italienische Recht, 2. Auflage, München 2008, S. 38 f., dort auch zur Sache. Ital. Originalfassung Gazzetta Ufficiale, 27 dicembre 1947, n. 298. 
breite Assoziationsebene eines „sozialen Rechtsstaats“ betreten. ${ }^{33}$ Fraglich sind die prinzipiellen Folgen für das Privatrecht. Aus deutscher Sicht fühlt man sich bei ,wirtschaftlicher Solidarität" an die Weimarer Verfassung erinnert, ${ }^{34}$ vor allem an Art. 151 Abs. 1 WRV:

„Die Ordnung des Wirtschaftslebens muß den Grundsätzen der Gerechtigkeit mit dem Ziele der Gewährleistung eines menschenwürdigen Daseins für alle entsprechen. In diesen Grenzen ist die wirtschaftliche Freiheit des Einzelnen zu sichern"

Anders als in der vom „Einzelnen“ ausgehenden Weimarer Verfassung, wird in Italien ein stärker solidaristischer Ton angeschlagen. Es geht nicht nur um individuelle Freiheit und deren Grenzen, sondern auch und gerade um deren Verwirklichung innerhalb gesellschaftlicher Entfaltungsräume, die auch als intermediäre Gewalten gedeutet werden können. Über Bindungswirkung und Tragweite dieser Kombination aus naturrechtlichem Freiheitspostulat und christlich-sozialer Semantik wurde lange gestritten. ${ }^{35}$ Mit der Zeit entwickelte sich daraus aber ein zentraler Anknüpfungspunkt für verfassungsrechtliche Überformungen italienischen Privatrechts. In der Rechtsprechung finden sich zahlreiche Fälle, in denen das verfassungsrechtliche Solidaritätsgebot als Korrektiv für Privatautonomie verwendet wurde. ${ }^{36}$ Eine Fallgruppe, in der die Prägung durch Art. 2 der Verfassung besonders deutlich ist, bezieht sich auf die Reduktion von Vertragsstrafen. ${ }^{37}$ Der italienische Codice civile enthält, wie BGB und Code civil, eine Norm, die eine richterliche Kontrolle bei exzessiven Vertragsstrafen erlaubt, nämlich Art. 1384 C.c.:

Die Vertragsstrafe darf vom Richter nach billigem Ermessen (equamente) reduziert werden, wenn die Hauptleistung teilweise erfüllt wurde oder der Betrag der Vertragsstrafe offensichtlich exzessiv ist, stets unter Berücksichtigung des Erfüllungsinteresses des Gläubigers.

Das gilt auch für Vertragsstrafen außerhalb des Verbraucherschutzrechts. ${ }^{38}$ Bei Verbraucherverträgen (contrattazione consumeristica) regelt Art. 33 f. des Verbrauchergesetzbuchs vom 2005 (Codice di consumo) eine von Amts wegen festzustellende Unwirksamkeit missbräuchlicher Klauseln (clausole vessatorie), wenn diese exzessive

33 Allgemein dazu Joachim Wege, Positives Recht und sozialer Wandel im demokratischen und sozialen Rechtsstaat, Berlin 1977, S. $204 \mathrm{ff}$.

$34 \mathrm{Zu}$ Solidarität in Weimar auch Uwe Volkmann, Solidarität - Programm und Prinzip der Verfassung, Tübingen 1997, S. 177-193.

35 Chantal Mak u.a, Abschnitt Italien, in: Brüggemeier u.a. (Hrsg.), Fundamental rights, Bd. 1, S. $330 \mathrm{f}$.

36 M.w.N. Andrea D 'Angelo/Pier Giuseppe Monateri/Alessandro Somma, Buona fede e giustizia contrattuale. Modelli cooperativi e modelli conflittuali a confronto, Turin 2005, S. 49, F. 54 .

37 Analyse dieser Rechtsprechung auch bei Mak, Fundamental rights, S. 110 ff. m.w.N.

38 Zur (Verbraucherschutz-) Rechtslage in Italien auch Mak u.a, Italy, in: Brüggemeier u.a. (Hrsg.), Fundamental rights, Bd. 1, S. 398 ff. 
Vertragsstrafen enthalten. ${ }^{39}$ Anders als in Deutschland, scheint das Recht der Allgemeinen Geschäftsbedingungen in Italien kein zentraler Anknüpfungspunkt für die Kontrolle von Vertragsstrafenklauseln zu sein. ${ }^{40}$ Hier gilt die tatbestandlich weniger ausdifferenzierte Klauselkontrolle nach Art. 1341 C.c. Bezüglich des Wortlauts von Art. 1384 C.c. fällt im Gegensatz zu seiner deutschen Parallelvorschrift § 343 BGB auf, dass im Codice civile keine Aussage darüber enthalten ist, ob es zur Reduktion der Vertragsstrafe eines Antrags des Schuldners bedarf, oder ob sie von Amts wegen möglich ist. Diese Frage der Vertragsstrafenkorrektur ex officio war Anlass von Debatten über eine „,costituzionalizzazione“441 (ein Begriff der italienischen Rechtsprechung) des Vertragsrechts.

Wie in Deutschland wegen $\S 253$ BGB, sind auch in Italien Bauträgerverträge ein praktisch wichtiges Anwendungsgebiet von Vertragsstrafenklauseln. Ausgelöst wurde die Debatte über Vertragsfreiheit und Grundrechte von einer Entscheidung aus dem Jahr 1999. ${ }^{42}$ In einem Vertrag über die Errichtung eines Hauses war ein Termin zur mangelfreien Fertigstellung vereinbart worden. Bei dessen Überschreiten sollte pro Tag eine bestimmte Strafsumme vom Bauunternehmer an den Käufer gezahlt werden, mit Ausnahmen von „höherer Gewalt“" und Vereitelung der Fertigstellung durch den Gläubiger. Zwei Monate nach dem Fertigstellungstermin zog der Käufer in das Haus ein, rügte aber Baumängel. Acht Monate später klagte er auf Zahlung einer Vertragsstrafe, die deutlich über dem vereinbarten Kaufpreis gelegen hätte, mit der Begründung, das Haus sei nicht vertragsgemäß errichtet worden. Der Beklagte stellte keinen Antrag auf Reduktion der Vertragsstrafe.

Bis 1999 hatte die Corte di Cassazione eine Herabsetzung von Amts wegen in derartigen Fällen abgelehnt, wie es auch dem deutschen Verständnis der Dispositionsmöglichkeiten und -pflichten des Schuldners bezüglich der Vertragsstrafe entspricht. ${ }^{43} 1999$ erfolgte die Kehrtwende. Begründung: Das verfassungsrechtliche Solidaritätsgebot müsse hier zu einer Einschränkung der Vertragsfreiheit führen. ${ }^{44}$ Der Rechtsstreit wurde somit an das Instanzgericht zurückverwiesen, mit der Aufgabe, die Vertragsstrafe zu reduzieren, ,ad equità“ ${ }^{45}$ also auf ein „,billiges Maß“. Auffällig dabei ist die schnelle Verlagerung der Argumentation auf eine verfassungsrechtliche Ebene. Man hätte auch zuerst privatrechtliche Instrumente diskutieren können. Vertragsfreiheit ist im Codice Civile als Prinzip verankert (Art. 1322 C.c.), jedoch natürlich nicht schrankenlos. Auch in Italien gibt es den Begriff der Sittenwidrigkeit, eingeführt über die Figur der causa des Vertrags. Diese darf nicht gegen gute Sitten verstoßen (Art. 1343 C.c.). Aber darum

39 Art. 33: (1) Im Vertrag zwischen Verbraucher und Unternehmer (professionista) werden Klauseln als missbräuchlich angesehen, die, wider Treu und Glauben, zu Lasten des Verbrauchers ein signifikantes Ungleichgewicht der vertraglichen Rechte und Pflichten festsetzen. (2) Als missbräuchlich gelten bis zum Gegenbeweis Klauseln, deren Wirkung oder Gegenstand darin besteht, (...) f. dem Verbraucher im Falle der Nichterfüllung oder verspäteten Erfüllung eine Geldsumme als Entschädigung, Strafklausel oder dergleichen von offensichtlich exzessiven Ausmaßen aufzuerlegen.

40 Anders in Deutschland wegen § 309 Nr. 6 BGB.

41 Corte di Cassazione n. 10511, 24. settembre 1999, Foro italiano, 2000, I, Sp. 1939.

42 Corte di Cassazione n. 10511, 24. settembre 1999, Foro italiano, 2000, I, Sp. 1929-1940.

43 Münch-Komm/Gottwald, 6. Aufl. 2012, §343 BGB, Rn. 12.

44 Corte di Cassazione n. 10511, 24. settembre 1999, Foro italiano, I, 2000, Sp. 1939.

45 Ebenda, Sp. 1940. 
ging es nicht. Hier sollte nicht eine im Einzelfall exzessive Strafe annulliert werden, was in Deutschland ja auch über $\S 138$ BGB möglich ist, sondern die richterliche Befugnis zum Eingriff in Verträge generell überprüft werden. Daher der Rückgriff auf „Solidarität“. Eingeleitet wird er durch ein historisches Argument: In modernen Rechtssystemen sei der aus dem 19. Jahrhundert stammende „Mythos der Omnipotenz des Willens" überwunden, die Unantastbarkeit der Verträge sei beendet, man gehe daher notwendigerweise von einer subjektiven zu einer objektiven und funktionalen Betrachtung der Vertragsstrafe über. ${ }^{46}$ Es gehe nicht nur um Eigeninteressen, die dem Schuldner zur selbständigen Disposition überlassen sind, sondern um die Anforderungen ,der Rechtsordnung“" im Allgemeinen. Das Modernisierungsargument öffnet den Weg in die Verfassung. Obwohl „Drittwirkung“ ein dem italienischen Rechtsdenken bekanntes Konzept ist ${ }^{47}$ und obwohl Kodifikation und Verfassung auch von dem hier erkennenden Gericht klar in den Stufenbau einer Normenhierarchie ${ }^{48}$ eingeordnet werden, macht die Rechtsprechung keinen Gebrauch davon. Vielmehr zitiert sie das Solidaritätsprinzip in gleichberechtigtem Zusammenspiel mit Generalklauseln der Kodifikation. ${ }^{49}$ Die Metaphorik des „Einfallstors“ und der „Ausstrahlung“ ist nicht explizit vorhanden, vielmehr geht es, so das Gericht wörtlich, um „Synergie“"50 zwischen Verfassung und Kodifikation, wobei deren unbestimmte Rechtsbegriffe von der Verfassung mit normativer Kraft aufgeladen und mit positivem Inhalt gefüllt wurden. Das alles führte zum Erfordernis einer Abwägung. Rechtsgut von Verfassungsrang ist in Italien nämlich nicht nur ,Solidarität" (Art. 2), wie das Gericht feststellt, sondern auch die private Initiative, wozu auch Vertragsfreiheit im Allgemeinen zählt (Art. 41). ${ }^{51}$ Erforderlich sei also eine Abwägung von „Werten“ (bilanciamento di valori) mit gleicher verfassungsrechtlicher Relevanz. ${ }^{52}$ Aus deutscher Sicht stellen sich Assoziationen zu Verhältnismäßigkeit und praktischer Konkordanz ein.

Diese Entscheidung des italienischen Kassationsgerichts blieb nicht ohne Widerspruch. Wie die Abwägung zu gestalten sei, wird nur vage gesagt, denn es geht dem Gericht vor allem um die Begründung der Notwendigkeit, also des $o b$, einer richterlichen Kontrolle der Vertragsstrafe, weniger um das wie der Ausführung, da ja dem Instanzgericht vorbehalten war. Nicht unterschieden wird daher zwischen verfassungsrechtlicher Werteabwägung und der Abwägung privater Interessen der Vertragsparteien, wie sie auch deutschen Beobachtern vertraut ist. Von Teilen der italienischen Literatur wurde hierin eine Gefährdung von Rechtssicherheit gesehen. ${ }^{53}$ Eine wichtige Konsequenz des verfassungsrechtlichen Solidaritätsgebots scheint zu sein, dass eine Semantik von

46 Ebenda, Sp. 1939.

47 Vgl. Hannah Nießen, Die Drittwirkung der Grundrechte in Deutschland und Italien, Hamburg 2005.

48 Corte di Cassazione n. 10511, 24. settembre 1999, Foro italiano, 2000, I, Sp. 1939. Es gehe um eine , rilettura degli istituti codicistici in senso conformativo ai precetti superiori della Costituzione Repubblicana“.

49 Ebenda.

50 Ebenda.

51 Art. 41 ital. Verfassung.: „Die wirtschaftliche Initiative ist frei. Sie kann nicht entgegen dem sozialen Nutzen ausgeübt werden, oder in einer Weise, die Beschädigungen von Sicherheit, Freiheit oder Menschenwürde verursacht.".

52 Corte di Cassazione n. 10511, 24. settembre 1999, Foro italiano, 2000, I, Sp. 1939.

53 Nachweise bei Mak, Fundamental rights, S. 114, m.w.N. 
Harmonie und Einklang der Parteiinteressen im Privatrecht entwickelt wurde. Wo Grundrechte gelten, scheint Harmonie wichtiger als prinzipielle Vertragsfreiheit und ihre Grenzen. In diese Richtung bewegt sich auch die Argumentation des Großen Senats (Sezione Unite), der im Jahr 2005 die Möglichkeit einer Reduktion von Vertragsstrafen von Amts wegen endgültig bestätigte, mit ausdrücklichem Verweis auf die Notwendigkeit vertraglichen Gleichgewichts. ${ }^{54}$ Freilich bewegt sich dessen Argumentation nicht nur auf verfassungsrechtlicher Ebene. Stärker abgestellt wird auf die bereits in der Kodifikation vorhandenen Schranken der Vertragsfreiheit ${ }^{55}$ und auf Vertragsauslegung. Der Große Senat setzt sich auch mit dem Argument auseinander, in der Zahlungsverweigerung des Schuldners könne man ohnehin einen Antrag auf Reduktion sehen. ${ }^{56}$ So wird Kritik am paternalistischen Eingreifen in Verträge durch eine weite Interpretation des Parteiwillens vorgebeugt. Maßgeblich blieb aber auch für den Großen Senat der Verweis auf das Verfassungsrecht. Auf methodischer Ebene eindeutig bleibt dabei die Notwendigkeit einer Abwägung von Interessen (congruo contemperamento degli interessi contrapposti). ${ }^{57}$ Methodisches Schlüsselwort ist contemperamento. Es bedeutet Anpassung und Harmonisierung zwischen Schuldner- und Gläubigerinteressen, aber auch zwischen Vertragsfreiheit und Solidarität. ${ }^{58}$

\section{Grundrechtsevolution: Vertragsfreiheit versus Berufsfreiheit Frankreich}

Werfen wir jetzt einen Blick auf ähnliche Phänomene in Frankreich. Dort hat eine bemerkenswerte „Konstitutionalisierung“ von Privatrecht im Bereich der nachvertraglichen Wettbewerbsverbote stattgefunden. Vertragsfreiheit stand hier im Gegensatz zu Berufsfreiheit. Die Ausgangsbedingungen sind hier jedoch grundverschieden im Vergleich zu Deutschland und Italien. Frankreich verfügt zwar seit 1789 über „fundamentale Prinzipien“, aber nicht über einen verbindlichen Grundrechtskatalog. Grundrechtspositionen können allerdings durch die Rechtsprechung des Conseil constitutionnel erzeugt werden. Solche spielten eine Rolle in Entscheidungen der Cour de Cassation bei abhängigen Arbeitsverhältnissen (durch die Chambre social) oder im Handelsvertreterrecht (durch die Chambre de commerce).

In den arbeitsrechtlichen Konstellationen ging es um die Frage der Zulässigkeit eines Wettbewerbsverbots mit oder ohne Karenzentschädigung. Vor 2002 war eine Karenzentschädigung nicht zwingend erforderlich. ${ }^{59}$ Dann kam das Revirement. ${ }^{60}$ Im Ausgangsfall waren drei Angestellte eines Frachtunternehmens zur Konkurrenz gewechselt. Ihre Verträge sahen Wettbewerbsverbote ohne Entschädigung vor. In den ersten Instanzen wurden die Angestellten zu einer ,indemnité“ an den alten Arbeitgeber verur-

54 Es gehe um „ricostruzione dell 'equilibrio contrattuale“, Corte di Cassazione, Sezione unite, n. 18128, 13 settembre 2005, Foro italiano, 2005, I, Sp. 2985 ff. (2988).

55 Ebenda, Sp. 2991.

56 Ebenda, Sp. 2988.

57 Ebenda, Sp. 2992.

58 Wie fest diese Figur mittlerweile im italienischen Vertragsrecht verankert ist zeigt auch eine weitere Entscheidung Corte di Cassazione, Sezione unite, 15 novembre 2007, n. 23726.

59 Zur älteren Rechtsprechung Dalloz 1998, S. 213-224.

60 Cass. Soc. 10. julliet 2002, Dalloz 2002, S. 2491. 
teilt. Das Kassationsgericht hob die Urteile jedoch auf; Begründung: die Klausel müsse mit dem ,principe fondamental“ der Berufsfreiheit vereinbar sein und mit Art. 120-2 des Code du travail. Dieser erlaubt ganz allgemein Beschränkungen individueller oder kollektiver Freiheitsrechte nur, wenn die Mittel im Verhältnis zum angestrebten Zweck stehen. ${ }^{61}$ Damit war ein fundamentales Prinzip mit einem einfachgesetzlichen Verhältnismäßigkeitsgebot verknüpft. Das Kassationsgericht wendete diese Kombination in einer Reihe von weiteren Fällen an und entwickelte daraus einen abschließenden Kata$\log$ kumulativ erforderlicher Kriterien für Wettbewerbsverbote: ${ }^{62}$

(1) Die Verbotsklausel muss demnach zur Verfolgung eines legitimen Interesses des Unternehmens erforderlich sein,

(2) sie muss zeitlich und räumlich begrenzt sein,

(3) die spezifischen Bedingungen des Arbeitsverhältnisses berücksichtigen und

(4) eine Karenzentschädigung (contrepartie financière) enthalten. Wenn letztere fehlt ist die Klausel von vornherein unwirksam, bei zu großer zeitlicher und räumlicher Ausdehnung sind geltungserhaltende Reduktionen durch den Richter möglich. ${ }^{63}$

Von der Literatur wurden diese Entscheidungen als „Tsunami“ im Recht der Wettbewerbsverbote bezeichnet. ${ }^{64}$ Ausführliche Begründungen findet man freilich in den Urteilen der Cour de Cassation nicht, die bekanntlich einem rituell anmutenden Sprachmuster folgen. Eher apodiktisch wirken die mit „Attendu que“ beginnen Satzkonstruktionen, die als stilistischer Legitimitätsfakor an sich weniger Raum für Analysen lassen und alles auf ein Grundprinzip (Berufsfreiheit) und dessen Kollision mit Interessen aus der Sphäre des Unternehmen argumentativ zuspitzen.

Wichtig sind für die eingangs beschriebene Fragestellung nun vor allem zwei Punkte: Erstens, worin der Grundrechtscharakter solcher Entscheidungen liegt und zweitens, wie die Kollisions- und Konfliktsituation des Grundrechts mit der Vertragsfreiheit gelöst wird. Bezüglich der ersten Frage scheint für den deutschen Beobachter auf der Hand zu liegen: Es geht um Berufsfreiheit (libre exercice d'une activité professionelle), also ein Grundrecht. ${ }^{65}$ Die französischen Richter reden von Berufsfreiheit als ,,principe fondamental“66 manchmal, aber wohl seltener, von ,principe constitutionel“ ${ }^{67}$. Woher kommen diese Prinzipien? Insgesamt ist in Frankreich eine Tendenz zu beobachten, Grundrechte aus der EMRK direkt im Privatrecht anzuwenden. ${ }^{68}$ Das ist aber eher beim Per-

61 Art. 120-2 Code du travail: Nul ne peut apporter aux droits des personnes et aux libertés individuelles et collectives de restrictions qui ne seraient pas justifiées par la nature de la tâche à accomplir ni proportionnées au but recherché.

62 Siehe die Urteile in Dalloz 2002, S. 2491, m. Anm. Yves Serra, S. 2492-2498. Generell Stefan Edenfeld, Nachvertragliche Wettbewerbsverbote im Europäischen Vergleich, ZfA 2004, $463 \mathrm{ff}$. (479).

63 Cass. Soc. 18. sept. 2002, Dalloz 2002, S. 3229.

64 Dalloz 2002, S. 2491.

65 Cass. Soc. 10. julliet 2002, Dalloz 2002, S. 2491. Zur verfassungsrechtlichen Dimension der neuen Rechtsprechung: Louis-Frédéric Pignarre, Clause de non-concurrence et droits fondamentaux $=$ Anm. zu Cass. Soc. 10. décembre 2008, Dalloz 2009, S. 1256.

66 Ebenda.

67 Hinweise bei Yves Serra, Note, Dalloz 2002, S. 2492, Fn. 2, siehe eine Entscheidung des Conseil Constitutionel zur Berufsfreiheit in AJDA 1983, S. 619.

68 Christoph Hermann/Chiara Perfumi, France, in: Brüggemeier u.a. (Hrsg.), Fundamental rights, Bd. 1, S. $201 \mathrm{f}, 205 \mathrm{f}$. 
sönlichkeitsrecht der Fall und hat vor allem deliktsrechtliche Auswirkungen. ${ }^{69}$ Auch finden sich Zivilurteile, die eine „liberté du travail“" aus der Präambel der Verfassung von 1946 ableiten, oder gar aus völkerrechtlichen Verträgen. ${ }^{70}$ Als Ergebnis lässt sich festhalten: Rückbindungen an höherrangige Normkomplexe gibt es zwar, sie wirken aber fast austauschbar. Die Bindung eine bestimmte Verfassung ist weniger wichtig. Prinzipien scheinen eher in der Rechtsordnung vorhanden zu sein und bedürften nicht unbedingt einer Verstärkung durch die Verfassung. Ein Zusammenspiel mit unbestimmten Rechtsbegriffen, wie man es für Italien gesehen hat, scheint ebenfalls weniger wichtig.

Die zweite Frage betrifft den Konflikt zwischen ,principe fondamental“ und Vertragsfreiheit. Gelöst wird er durch einen Abwägungsvorgang, die französische Doktrin spricht hier von „,conciliation“71 zweier Bedingungen (,conditions“) ${ }^{72}$ und zwar nicht der Vertrags- und der Berufsfreiheit, sondern meist konkreter, vom legitimem Unternehmensinteresse und der Berufsfreiheit. Zentrales Legitimationsmittel für die Beschränkung der Vertragsfreiheit ist die Art und Weise dieser concilitaion, hier wie gesagt ausgerichtet an einer dem Code du travail entnommenen ${ }^{73}$ Zweck-Mittel-Prüfung. Aber auch über diese Fälle hinaus hat das Kriterium der Verhältnismäßigkeit (proportionalité) in der französischen Doktrin in letzter Zeit Aufmerksamkeit erfahren. Zweck-Mittel-Relationen werden nicht nur bei Berufsfreiheit auf arbeitsrechtlicher und handelsrechtlicher Ebene thematisiert, sondern auch bei anderen Rechtspositionen, wie dem Persönlichkeitsrecht gem. Art. 9 Code civil (oft im Zusammenhang zitiert mit den entsprechenden Vorschriften aus der EMRK, Art. 8 und 10). ${ }^{74}$ Persönlichkeitsrecht und Informationsfreiheit, so die Cour de Cassation, seien gleichwertig (identique valeur normative), daher habe der Richter beide Rechte in Ausgleich zu bringen (rechercher leur equilibre). ${ }^{75}$ Das geschehe durch Privilegierung der Lösung, die das Interesse mit dem höchsten Legitimitätsgrad am besten schütze. ${ }^{76}$ Die Literatur stellt diese Wertung in den Zusammenhang der französischen Methodengeschichte. Sie sei eine schöne Hommage an den Doyen Gény - gemeint ist Francois Gény, der in seiner „Methode d interpretation“ (erste Auflage 1899) einen gerechten Ausgleich verschiedener Positionen (juste conciliation) als Anspruch verkündete, der schließlich auf die Verwirklichung eines ,fin social de l'humanité“ hinauslaufen sollte. ${ }^{77}$ Die Literatur macht daraus

69 Siehe Art. 8 und 10 EMRK, dazu: Jean Hauser, Droits de la personnalité, in: RTD civ. 2003, 680. Und das, obwohl das Persönlichkeitsrecht selbst in Art. 6 Code civil verankert ist, man also direkt auf die Kodifikation zurückgreifen kann.

70 So in einem Urteil über Teilzeitarbeit, in: JCP, La semaine juridique, 2000, S. 1835, Nr. 2556 und Nr. 2557 (beide Cass.soc). Siehe auch einen Verweis auf die Berufsfreiheit im Sinne des „Pacte international relatif aux droits économiques, sociaux et culturels“"vom 16.12.1966, der als ,directement applicable en droit interne“ bezeichnet wird. Cass. Soc. 16. décembre 2008, Dalloz 2009, S. 233.

71 Abwägung kann man auch mit ,deliberation“ übersetzen.

72 So die Wortwahl bei Yves Serra, Note, Dalloz 2002, S. 3229.

73 So die Urteile Dalloz 2002, S. 2491.

74 Cass. Civ. 9. juilliet 2003, Dalloz 2004, S. 1633 f. mit Anmerkung v. Christophe Caron.

75 Ebenda, S. 1634.

76 Ebenda.

77 Francois Gény, Méthode d interpretation et sources en droit positif, Bd. 2, 2. Aufl. Paris 1919, S. 167. Verweis auf Gény bei Christope Caron, Dalloz 2004, 1634. 
eine „balance des interets“. Sehr ähnlich verhält es sich auch bei der Berufsfreiheit im Rahmen des Wettbewerbsverbots.

Beobachten lässt sich also eine klare „Konstitutionalisierung“. Vertragsfreiheit wird Grundlage potentieller Eingriffe in die Berufsfreiheit, welche am Maßstab der „Verhältnismäßigkeit“" gemessen werden. Endziel bleiben - wie in der Rechtsprechung der italienischen Corte di Cassazione - Harmonie und Ausgleich zwischen den Konsequenzen von Vertragsfreiheit und ihren Schranken. Es geht weniger um allgemeine zivilrechtliche Verhältnismäßigkeitsbegriffe, ${ }^{78}$ sondern durchaus um Dinge, die man aus deutscher Sicht mit praktischer Konkordanz assoziiert. Widerstreitende Prinzipien stehen also im Privatrecht grundsätzlich auf einer Stufe, wohl auch die Vertragsfreiheit und ihre möglichen fundamentalrechtlichen Beschränkungen.

\section{Vergleich der Konstitutionalisierungsprozesse in Italien und Frankreich}

Im Vergleich kann man nun fünf Ergebnisse beobachten:

(1) In Italien und Frankreich spielen Konstitutionalisierungsprozesse von Vertragsrecht seit längerem eine Rolle, wobei in Italien die Angriffspunkte der Verfassung im Vertragsrecht zahlreicher sind und die Rechtsprechung wohl früher auf Grundrechte zurückgegriffen hat.

(2) Sehr unterschiedlich sind die Referenzpunkte der Konstitutionalisierung. In Italien ist eindeutig der nationale Grundrechtskatalog zentraler Ausgangspunkt einer Überformung des Vertragsrechts. Supranationale Rechte spielen kaum eine Rolle. In Frankreich wird hingegen mit einem sehr offenen Grundrechtsbegriff operiert, dessen Rückbindung an eine bestimmte Verfassung zweitrangig zu sein scheint.

(3) In beiden Ländern hat die fundamentalrechtliche Einkleidung von Vertragsrecht ähnliche methodische Konsequenzen. Sie führt zu einer Abwägung - doch wovon? Manchmal ist von Interessenabwägung die Rede, welche durch Grundrechte indiziert wird (so eher in Frankreich), in Italien werden auch Grundrechtspositionen auch direkt Privaten zugewiesen, abgewogen werden „Interessen“, „Werte“ (valori) oder schlicht „Vertragsfreiheit“ versus „Solidarität“, wobei letzteres als Beschränkung des ersteren aufzufassen ist.

(4) Die Abwägung geschieht in Italien nicht anhand konsolidierter Methoden, sondern eher freihändig. In Frankreich hingegen sind kanonisierte Zweck-Mittel-Prüfungen im Vordringen, unter dem Stichwort ,proportionalité“, und das nicht nur im Arbeitsrecht, wo das Gesetz einen Anknüpfungspunkt für diese Rechtsfigur zur Verfügung stellt.

(5) Unterschiede gibt es auch beim Einsatz von Generalklauseln der Kodifikation. In Italien sind sie wichtig und wirken mit den Grundrechten zusammen. In Frankreich scheint die Idee der Generalklauselkonkretisierung mit Hilfe von Grundrechten weniger wichtig zu sein.

78 Zur Abgrenzung Dieter Medicus, Verhältnismäßigkeit im Zivilrecht, AcP 192 (1992), S. 36-70. 


\section{Interpretationen}

Manche der Unterschiede könnten im Zusammenhang rechtskultureller Bedingungen erklärbar sein. Zu überlegen wäre etwa, ob in Frankreich ein Verständnis des Code civil als Ausdruck und Grundlage einer revolutionär erkämpften Gesellschaftsordnung eine Eigendynamik des Privatrechts als Prinzipienordnung mit Menschenrechtsqualität begünstigte, die dem BGB und dem Codice civile kaum zugeschrieben wurde. Das ist eine große Frage, die grundlegende Langzeitbeobachtungen zum Verhältnis von Privatrecht und Verfassung voraussetzt. Solche sind vor allem den Studien von Dieter Grimm zu entnehmen. ${ }^{79}$ Dort werden verschiedene Grundmuster zur historischen Verhältnisbestimmung zwischen Grundrechten und Privatrecht beschrieben. Für Frankreich hebt Grimm die aktive Rolle der Grundrechte (in Gestalt der Déclaration des droits de l'homme et du citoyen von 1789) bei der revolutionären Verwirklichung einer bürgerlichen Sozialordnung hervor. ${ }^{80}$ Sie könnten wegen dieser Sonderrolle als Vorstufe eines bürgerlichen Privatrechts zu betrachten sein, zumal es in den Menschenrechtsdebatten der Nationalversammlung nicht nur um Garantien gegen den Staat, sondern um Grundsätze mit Geltungsanspruch für und zwischen allen Rechtssubjekten ging. ${ }^{81}$ Aktuell würde die Frage also lauten, ob man aus dieser revolutionären Ursprungssituation generell auf eine stärkere Durchdringung des französischen Privatrechts mit Grund- und Menschenrechtsprinzipien schließen kann. Wenn der Code Civil von 1804 eine Form von grundrechtsverwirklichender Gesetzgebung wäre, also zentraler Bestandteil eines aus Grundund Menschrechten herauswachsenden Rechts einer (in Wechselwirkung damit) zu bildenden bürgerlichen Gesellschaft, müsste einiges von der universellen Tragweite der Grundrechte auf die Kodifikation abgefärbt sein. Das würde dann die Grundsatzorientierung französischen Privatrechts plausibel erscheinen lassen und seine Indifferenz gegenüber Grenzdurchbrechungsfragen wie Drittwirkung und der Metaphorik von Ausstrahlung eines Rechtsbereichs in einen anderen erklären helfen, die etwa in Deutschland so wichtig sind.

Die Beobachtungen von Grimm stützen diese These jedoch kaum. Er kommt zu dem Ergebnis, dass die Grundrechte mit Vollendung der bürgerlichen Privatrechtsordnung auf eine Garantiefunktion von Rechten gegenüber dem Staat zurückgestuft worden seien, die im Zuge der Restauration besondere Bedeutung erlangt habe. ${ }^{82}$ Der Code Civil habe die ursprünglich enge Verbindung von Grundrechten und Privatrecht aufgelöst; es habe sich herausgestellt, dass Grundrechte „nur ein additionelles, kein essentielles Element“ eines „bürgerlichen Sozialmodells“ bildeten, dessen conditio sine qua non das Privatrecht sei. ${ }^{83}$ Grundrechte wurden nach dieser Geschichtsbetrachtung zum äußeren Schutzschirm der Privatrechtsordnung, gehörten aber nicht zu ihren zentralen Bestanteilen.

Für Deutschland ist hingegen die Fokussierung auf die Abwehrfunktion der Grundrechte historisch noch eindeutiger zu erklären. Grimm betont im Hinblick darauf, dass

79 Insbesondere Dieter Grimm, Grundrechte und Privatrecht in der bürgerlichen Sozialordnung, in: ders. Recht und Staat der bürgerlichen Gesellschaft, Frankfurt/M, 1987, S. 192-211.

80 Ebenda, S. 199.

81 Ebenda, S. 200.

82 Ebenda, S. 201.

83 Ebenda. 
im Grundrechte im deutschen Frühkonstitutionalismus von der Obrigkeit verliehene Rechte waren. ${ }^{84}$ Ohne naturrechtlich universelle Tendenz seien sie nur auf Schutz bestimmter Rechtspositionen, vor allem des Eigentums gerichtet gewesen. ${ }^{85}$ Ein Blick in entsprechende Verfassungen bestätigt das. ${ }^{86}$ Deutlich wird hier eine historische Trennungslinie zwischen Grundrechten und Privatrecht in Deutschland. ${ }^{87}$ Relativiert wurde diese vor allem nach dem Zweiten Weltkrieg, wo das Grundgesetz unter dem Eindruck des Unrechtsstaats als Werteordnung einen besonderen Rang eroberte. Mehr als in anderen Ländern war die Verfassung nach 1949 Projektionsfläche von Hoffnungen und Ausgangspunkt von Erwartungen. Für manche sollte sie gar zum neuen Identitätszentrum einer Rechtsordnung werden. So blieb zwar das Bewusstsein der Grundrechte als Abwehrrechte prägend, Ausstrahlungen ins Privatrecht waren wegen der Aufwertung der Verfassung aber leicht zu rechtfertigen. Diese Erfahrungen hat man in Frankreich nicht geteilt. Parallelen zu Italien scheinen eher möglich, müssten aber, wie dieses Thema überhaupt, mit historischen Analysen vertieft werden.

Offensichtlich ist insgesamt, dass die deutsche Perspektive der „Drittwirkung“ natürlich ein konsolidiertes Verständnis von Grundrechten als Abwehrrechte voraussetzt, das in anderen Ländern sehr verschieden sein kann. In Italien etwa deutet bei Art. 2 der Verfassung bereits der Wortlaut auf ein Hineinragen des Solidaritätsanspruchs in alle gesellschaftlichen Sphären, was zeigt, wie sehr die Drittwirkungsfrage mit einem deutschen Grundrechtsverständnis verhaftet ist, das sich auf andere Staaten nicht projizieren lässt.

\section{Auf dem Weg zu einer europäischen Abwägungs- oder Wertungsjuris- prudenz?}

Konstitutionalisierungsprozesse könnten in Zukunft generell wichtiger werden. Ein eher grundsatzorientiertes Privatrecht scheint im Vordringen zu sein, wenn man aktuelle Privatrechtskodifikationen betrachtet. So enthält etwa das neue rumänische Zivilgesetzbuch von 2009 in einem Anfangskapitel über „Allgemeine Vorschriften“ ein Bekenntnis zur Grundrechtbindung des Privatrechts. ${ }^{88}$ Dessen Normen müssten im Einklang ${ }^{89}$ mit der Verfassung, der Allgemeinen Erklärung der Menschenrechte, ${ }^{90}$ sowie

84 Ebenda, S. 203, wobei die Unterscheidung zwischen oktroyierter und nichtoktroyierter Verfassung aber nicht reflektiert ist.

85 Ebenda, S. 204.

86 Etwa Abschnitt II $\S \S 13,14$ der Verfassung Badens von 1818 und Abschnitt IV $\S 8$ der Verfassung Bayerns aus demselben Jahr, wo Eigentums- und Freiheitsrechte negativ-staatssauschließend und nicht als universelle Rechtspositionen formuliert sind.

87 Was aber nicht ausschließt, dass der Code civil zum „Grundrechtsersatz“ werden konnte, wo wie in den preußischen Rheinprovinzen eine Verfassung fehlte. Siehe Grimm, ebenda, S. $206 \mathrm{f}$.

88 Dazu Christiane Wendehorst, Methodennormen in kontinentaleuropäischen Kodifikationen, RabelsZ 75 (2011), S. 730-763 (747).

89 Anders jedoch Wendehorst, Methodennormen, S. 747, die von einem „Vorrang“ internationaler Menschenrechtsabkommen ausgeht, was sich nur unter Hinzuziehung der Überschrift des Artikels rechtfertigen lässt.

90 Gemeint ist an dieser Stelle die UN-Menschenrechtscharta von 1948, nicht die EMRK. 
internationalen Vereinbarungen, an denen Rumänien beteiligt ist, ausgelegt und angewendet werden. ${ }^{91}$ Das Recht der Europäischen Union soll Vorrang vor der nationalen Kodifikation haben. ${ }^{92}$ Konstitutionalisierung des Privatrechts gehört hier also zu den zentralen Bekenntnissen einer Kodifikation.

Noch deutlicher grundsatzbezogen ist ein 2001 vorgelegter Entwurf für eine tschechische Zivilkodifikation. Hier wird eine Art verfassungskonforme Auslegung des einfachen Gesetzes an am Anfang der Kodifikation postuliert. ${ }^{93}$ Das Privatrecht könne nur im „Einklang mit der Liste von Grundrechten und Freiheiten, dem Verfassungsrecht überhaupt, den Grundsätzen, auf denen das Gesetz aufbaut, sowie mit Rücksicht auf die Werte, die hiermit geschützt werden sollen, ausgelegt werden." ${ }^{94}$ Es folgen mehrere Normen in unverkennbarem Grundrechtston. Das Privatrecht schütze Freiheit, Menschenwürde und vor allem: Glücksstreben. ${ }^{95}$ Ein Grundrecht par excellence der Virginia Declaration of Rights von 1776, ${ }^{96}$ einer der Archetyen modernen Verfassungsrechts, steht hier an prominenter Stelle in einer Privatrechtskodifikation. Aufgezählt werden dann Rechtsgüter auf denen das Privatrecht beruht (Recht auf Leben, Gesundheit, Familie, Ehre, Würde, Privatsphäre etc.), aber auch Minderjährigenschutz. ${ }^{97}$ Von Vertragsfreiheit ist explizit nicht die Rede, nur von Freiheit allgemein, Versprechenstreue und pacta sunt servanda. ${ }^{98}$ Gegenüber den hehren allgemeinverständlich formulierten Grundsätzen, würde sich eine prinzipielle Absicherung individueller Beteiligungsspielräume am Rechtsverkehr im Sinne willensgestalteter Geschäftssphären trocken und technokratisch ausnehmen. Eigentumsfreiheit gehört zu den Grundsätzen des Zivilrechts, steht aber nicht mehr an erster Stelle. ${ }^{99}$ Der eigene Kräfte mobilisierende, durch Erwerbsstreben zu Glück und Freiheit gelangende Bürger lässt sich in diesen Passagen des Entwurfs zwar wiederfinden, ist aber längst nicht mehr prägende Hauptfigur. Worauf es ankommt ist „,der Mensch“ im sozialen Umfeld und seine absoluten Rechte. Eindeutiger kann der Anspruch einer Materialisierung von Privatrecht kaum hervortreten.

Die Frage nach den strukturellen Konsequenzen solcher Grundsatzorientierungen für die europäischen Privatrechtssysteme stellt sich also umso mehr, wenn im Zuge der Internationalisierung die Grenzen zwischen öffentlichem Recht und Privatrecht undeutlicher werden und europäische Grundrechte an Einfluss gewinnen. Bei aller Hete-

91 So Art. 4, auch der aktuellen Fassung des Gesetzbuchs von 2011, in: Monitorul oficial al Romanei, Partea I, Nr. 505/15.VII.2011.

92 Ebenda, Art. 5.

93 Wendehorst, Methodennormen, S. 748.

94 Entwurf § 2, in deutscher Übersetzung zitiert nach Wendehorst, Methodennormen, S. $748 \mathrm{f}$.

95 Entwurf \& 3, ebenda.

96 ,A Declaration of Rights made by the representatives of the good people of Virginia, assembled in full and free Convention; which rights do pertain to them, and theier posterity, as the basis and foundation of government. I. That all men are by nature equally free and independent, and have certain inherent rights, of which, when they enter into a state of society, they cannot, by any compact, deprive or divest their posterity; namely, the enjoyment of life and liberty, with the means of acquiring and possessing property, and pursuing and obtaining happiness and safety." Zitiert nach Robert Allen Rutland, The Birth of the Bill of Rights 1776-1791, Chapel Hill 1955, S. 231.

97 Entwurf § 3, in deutscher Übersetzung zitiert nach Wendehorst, Methodennormen, S. 748.

98 Entwurf § 3 (2) d), ebenda, S. 749.

99 Entwurf $\S 3$ (2) e), ebenda, S. 749. 
rogenität der so möglicherweise voranschreitenden Konstitutionalisierungsprozesse scheint eine Gemeinsamkeit unübersehbar: An Stelle des Schemas prinzipieller Vertragsfreiheit und deren Begrenzung mit zivilrechtlichen Schranken tritt ein Konflikt zwischen Rechtsgütern, was zu einem Bedeutungszuwachs von Abwägungsvorgängen führt. ${ }^{100}$ Dieser wird kontrovers beurteilt. Grundrechtspessimisten fürchten einen Bedeutungsverlust der Vertragsfreiheit und einen Verlust von Rechtssicherheit. Andere stehen vor allem der Abwägung als Methode skeptisch gegenüber, da sie eine Auflösung rechtsstaatlich gebotener Normenstrenge in Einzelfalljurisprudenz befürchten. ${ }^{101}$ Grundrechtsoptimisten sehen höherrangiges Recht hingegen als normative Kraftquelle zur Aufladung des Privatrechts mit sozialer Energie, die gerade in einer auf (wirtschaftlichen) Grundfreiheiten aufgebauten Europäischen Union für nötig gehalten wird. ${ }^{102}$ Gleichzeitig trauen sie den konstitutionalisierten Rechtsfindungen zu, die grundsätzliche Dimension von Problemen aufscheinen zu lassen. Demzufolge betrachten sie Grundrechte im Privatrecht als Indikatoren für politische Konflikte im Privatrecht, von dem sie befürchten, es könne ein dem politischen Alltag entrücktes Normengebilde mit intransparenten Autoritätsansprüchen werden. Die Frage ist aber, ob Grundrechte im Privatrecht diesen Anspruch einlösen, oder vielleicht sogar das Gegenteil bewirken können. Nicht auszuschließen ist, dass Gerichte in grundrechtsorientierten Entscheidungen mit Einzelfall- und Harmonierhetorik die Befürwortung einer bestimmten Option verschleiern, dass sie die Privilegierung bestimmter Lösungen, Werte oder Interessen nicht einräumen. Im schlimmsten Fall könnten vielleicht sogar bestimmte gesellschaftliche Gruppen bevorzugt werden, von Entscheidungsträgern, die sich dabei als Verfechter einer höheren, objektiven Ordnung gerieren. Wenn an „Solidarität“ appelliert wird, kann in Wirklichkeit auch die Baubranche gemeint sein, die man vor Gewinneinbußen durch Vertragsstrafen bewahren will. Als politisches Ziel mag das legitim sein, es wäre aber auf jeden Fall transparent zu machen - ganz abgesehen von der Frage, ob die Judikative dafür zuständig ist. Hinter der angeblichen „Auflösung“ eines Rechtskonflikts, der „Harmonie“ von Interessen, verbirgt sich in der Regel für eine Partei eine Niederlage, die vielleicht nur von einem Scheinfrieden überdeckt wird.

\section{Ausblick: Die Rolle von Grundrechten in einer europäischen Metho- denlehre}

Insgesamt können solche rechtspolitischen Fragen nur mit einem Blick auf die funktionalen Äquivalente der Grundrechte beantwortet werden. In Frankreich ist Grenze der Vertragsfreiheit der „ordre public“(Art. 6), in Italien der „ordine pubblico“ (Art. 1343)

100 Vgl. die Schlussfolgerung der umfangreichen Projektanalyse von Aurelia Colombi Ciacchi, in: Brüggemeier u.a. (Hrsg.), Fundamental rights, Bd. 2, S. $431 \mathrm{ff}$.

101 Joachim Rückert, Abwägung - die juristische Karriere eines unjuristischen Begriffs oder: Normenstrenge und Abwägung im Funktionswandel, JZ 2011, S. 913-923 (922).

102 Aus französischer Sicht etwa Chrisophe Jamin, Le droit des contrats saisi par les droits fondamentaux, in: Gegory Lewkowitz/Mikhail Xiafras (Hrsg.), Repenser le contrat, Paris 2009, S. 173-259 (177). Hier ist metaphorisch von ,irrigation“ also Bewässerung (des Privatrechtsgartens) durch Grundrechte die Rede, die dort eine „Idee du social“" entstehen lassen könnten. 
daneben ermöglicht die cause oder causa-Lehre vertragliche Legitimitätskontrollen. Wie diese ähnlichen Begriffe in der Rechtsprechung konkretisiert werden, ist eine zentrale Frage für einen Vergleich. Sicher ist zumindest, dass in den Verweisen auf öffentliche Ordnung eher ,objektive“ Interessen angesprochen sind, wie der Schutz staatlicher Institutionen. Die Grundrechtsordnung erlaubt es immerhin, subjektive, individuelle Rechte mit der Vertragsfreiheit auf Kollisionskurs zu bringen. Das wäre etwa im Beispiel der Berufsfreiheit mit dem französischen ordre public nicht ohne weiteres möglich gewesen. Auch die italienische causa-Lehre hebt eher auf die Legitimität einer ökonomisch-sozialen Funktion von Rechtsgeschäften ab, weniger auf Individualrechte. ${ }^{103}$ Richterliche Fortbildung in dieser Richtung erscheint aber nicht ausgeschlossen.

Wenn solche klassischen Wege der Beschränkung von Vertragsfreiheit verlassen werden, stellt sich die Frage, was das methodisch bedeutet. Wie gesagt führt es zu einer Vermehrung von „Abwägungsvorgängen“ und die sind - zumindest nach deutschem Verständnis - einzelfallbezogen. Eine Rechtsfortbildung entlang der Schranken der Kodifikationen beansprucht normative Konsolidierung über den Einzelfall hinaus. Wenn die Alternative zur (grundrechtlich indizierten) Abwägung Regelbildung anhand von Fallgruppen sein soll, ${ }^{104}$ dann ist zu überprüfen, welche Rolle Fallgruppen im tradierten Instrumentarium eines Rechtssystems spielen. Die Frage lautet, wie Fallgruppen gebildet und durch welche Medien sie kommuniziert werden. Offensichtlich ist, dass die Suche nach funktionalen Äquivalenten zur Beschränkung der Vertragsfreiheit durch Grundrechte nicht ohne eine vergleichende Analyse klassischer Rechtsfortbildungs- und Auslegungsmethoden erfolgen kann. Notwendig für wertende Aussagen über Grundrechtswirkungen in Europa ist also auch eine Verbindung mit den Ergebnissen europäischer Methodenvergleiche. ${ }^{105}$ So könnte man genauer beobachten, wie sich Grundrechtsanwendungen und Einzelfallabwägungen zu Regeln verfestigen können. ${ }^{106}$ Dass so etwas möglich ist, hat das vorhin zitierte Beispiel der französischen Rechtsprechung über die Wettbewerbsverbote gezeigt, bei der von der grundrechtsgeneigten Lösung im Einzelfall sofort - nicht erst von der Doktrin, sondern bereits von der Cour de Cassation selbst - zu allgemeiner Regelbildung abstrahiert wurde. Kehrt man zum Beispiel der Vertragsstrafe zurück, so zeigt auch ein Blick auf die deutsche Rechtsprechung, wie sich Abwägungen zu Regeln verdichten können. In Deutschland ist bekanntlich die Vertragsstrafe, wie bereits gesagt, ebenfalls bei Bauleistungen relevant, genau wie in Italien. ${ }^{107}$ Angemessenheit wurde vom Bundesgerichtshof ebenfalls aufgrund von Interessenabwägungen geprüft, allerdings vornehmlich im Recht der Allgemeinen Geschäftsbedingungen ( $\$ 307$ BGB). Hier handelte es sich um Einzelfalljudikatur in Ausfüllung einer Generalklausel, die dennoch gewisse Standards für die Baubranche richterrechtliche typisierte. Zur Absicherung von Bauleistungen ist seit einer Konkretisie-

103 Nachweise bei Kindler, Einführung, S. 129 f.

104 Ausführlich Rückert, JZ 2011, S. 922.

105 Aktuelle Ansätze wurden vor allem auf einem Symposion „Europäische Methodenlehre“ 2010 in Hamburg erörtert. Dazu Holger Fleischer, Europäische Methodenlehre: Stand und Perspektiven, RabelsZ 75 (2011), S. 700-729.

$106 \mathrm{Zu}$ Ergebnissen von Abwägungsvorgängen bereits Aurelia Colombi Ciacchi, in: Brüggemeier u.a. (Hrsg.), Fundamental rights, Bd. 2, S. $431 \mathrm{ff}$.

107 Gem. Art. 2059 C.c. können Nichtvermögensschäden in Italien nur in den gesetzlich vorgesehenen Fällen ersetzt werden. Es gibt also eine Parallelnorm zu § 253 BGB. 
rung der Rechtsprechung im Jahr $2003^{108}$ nur noch eine Vertragsstrafe von bis zu $5 \%$ der Auftragssumme angemessen. ${ }^{109}$ Hier haben also Interessenabwägungen und Schranken der Vertragsfreiheit in einer Generalklausel zusammengewirkt. Eine Einzelfallabwägung mit oder ohne Grundrechte geht nicht immer mit einem dauerhaften Verlust an Rechtssicherheit einher. Das zeigt: Die Einführung von Grundrechten ins Privatrecht muss nicht immer einen Tsunami auslösen, auch nicht auf methodischer Ebene. Um solche Fragen zu vertiefen, sind Ansätze der vergleichenden Rechtsprechungsanalyse weiterzuverfolgen und auf andere Länder auszudehnen. So könnten weitere Erkenntnisse über die dogmatischen Implikationen einer Materialisierung des Privatrechts gewonnen werden. Gleichzeitig könnte mit solchen Erkenntnissen auch die politische Debatte um ein soziales Privatrecht für Europa bereichert werden, für das die richterliche Rechtsfortbildung ein zentraler Faktor ist. Insgesamt ist es daher für die Zukunft notwendig, die spezifische Rolle von Grundrechten im Rahmen einer europäischen Methodenlehre ${ }^{110} \mathrm{zu}$ reflektieren, unabhängig davon, ob man diese als Vorstufe einer in Zukunft zu verwirklichenden Rechtseinheit ansieht, oder als Methode eines geltenden „Unionsprivatrechts“. ${ }^{111}$ Es bedarf einer eingehenden Betrachtung der Rolle von Verfassungsgrundsätzen in Methodenlehren einzelner europäischer Privatrechtssysteme. Dabei ist es unverzichtbar, den methodischen Erfahrungsschatz europäischer Rechtssysteme mit historisch-vergleichenden Analysen freizulegen. Besonders vielversprechend ist dabei der Blick auf die Rechtsprechung, in der sich die Methoden spiegeln.

108 BGH, NJW 2003, S. 1806.

109 Zuletzt dazu OLG-Brandenburg Urteil vom 12.10.2011 - 13 U 86/07.

110 Literaturnachweise bei Fleischer, RabelsZ 78 (2011), S. 701.

$111 \mathrm{Zu}$ verschiedenen Funktionen einer europäischen Methodenlehre Fleischer, ebenda, S. $710 \mathrm{ff}$. 